\title{
Exploring digital image correlation technique for the analysis of the tensile properties of all-cellulose composites
}

\author{
Feng Chen · Jean-Luc Bouvard • Daisuke Sawada $\cdot$ Christophe Pradille • \\ Michael Hummel $\cdot$ Herbert Sixta $\cdot$ Tatiana Budtova $(\mathbb{C}$
}

Received: 3 November 2020/Accepted: 3 March 2021 / Published online: 20 March 2021

(C) The Author(s) 2021

\begin{abstract}
All-cellulose composites (ACCs) were prepared from filter paper via partial dissolution in the ionic liquid 1-ethyl-3-methylimidazolium acetate, and material tensile properties were investigated using various approaches. One is based on data directly taken from a tensile testing machine, and the other uses two-cameras stereovision with digital image correlation (DIC) technique. In the latter case, virtual extensometer with different locations on the sample and averaging over sample surface were tested. Nominal and true stress-strain dependences were built and Young's modulus, tensile strength, elongation at maximal stress and toughness were evaluated as
\end{abstract}

Supplementary Information The online version contains supplementary material available at https://doi.org/10.1007/ s10570-021-03807-9.

F. Chen · D. Sawada - M. Hummel .

H. Sixta - T. Budtova

Department of Bioproducts and Biosystems, School of Chemical Engineering, Aalto University, P.O. Box 16300, 00076 Aalto, Helsinki, Finland

J.-L. Bouvard · T. Budtova ( $₫)$

MINES ParisTech, PSL Research University, Center for Materials Forming-CEMEF, UMR CNRS 7635, CS

10207, 06904 Sophia Antipolis, France

e-mail: tatiana.budtova@mines-paristech.fr

C. Pradille

Mat Xper, 19 Traverse du Barri, 06560 Sophia Antipolis, France a function of ACC density. A minor difference was observed for the stress-strain dependences derived from different approaches which use the DIC technique, most probably because of low ACC deformation. However, the results reveal that the nominal stress-strain curve from DIC is significantly different from that which is directly derived from the data provided by machine sensors thus strongly impacting Young's modulus and elongation at break values. This study provides an insight into the evaluation of the mechanical properties of ACCs. 


\section{Graphic abstract}

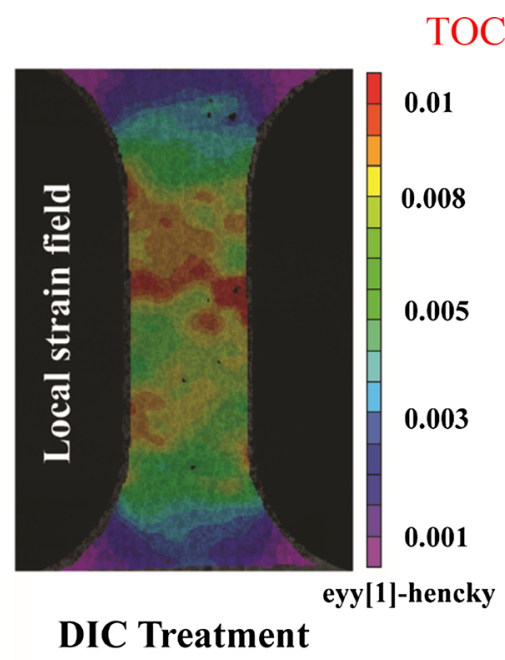

Keywords Filter paper - Ionic liquid · Tensile properties $\cdot$ Digital image correlation

\section{Introduction}

The growing concerns on environmental issues motivate the development of biobased and biodegradable materials such as biocomposites. One of the subclasses of biocomposites is all-cellulose composites (ACCs) (Nishino et al. 2004). The principle of ACC fabrication is based on the concept of all-polymer composites in which the matrix and the reinforcing fibers are made from the same matter (Capiati and Porter 1975). The advantage of this concept is that the adhesion between the matrix and the fibers is perfect, and there is no need of compatibilisers. The difference between all-polymer and all-cellulose composites is that cellulose is not melting, and thus ACC preparation requires a dissolution step followed by washing out the solvent and drying.

There are two main routes for making all-cellulose composites: (1) one-step method via partial dissolution of fibers in cellulose solvent with the dissolved cellulose forming the composite matrix (Soykeabkaew et al. 2008; Huber et al. 2012; Piltonen et al. 2016; Khakalo et al. 2019; Chen et al. 2020a) and (2) twostep method which involves the preparation of a cellulose solution used for the impregnation of the reinforcing fibers (Nishino et al. 2004; Spörl et al.

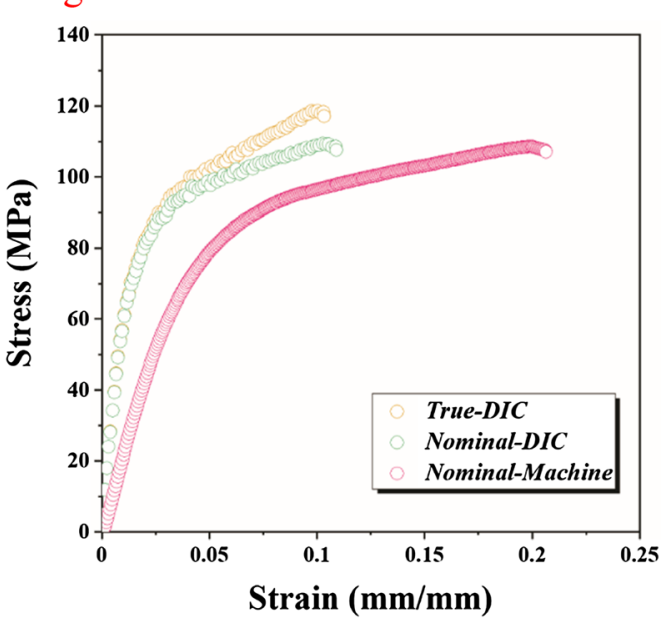

2017; Labidi et al. 2019; Korhonen et al. 2019). In both cases the reinforcing phase can be natural or manmade fibers, aligned or isotropic (dispersed short fibers, a fabric, or a filter paper). Nanocellulose based composites will not be considered here as it is out of the scope of this work.

Various cellulose solvents have been used to make ACCs: LiCl/dimethylacetamide (DMAc) (Nishino et al. 2004; Soykeabkaew et al. 2008), NaOH-water based solutions (Piltonen et al.2016; Korhonen et al. 2019) and, recently, ionic liquids (Spörl et al. 2017; Khakalo et al. 2019; Chen et al. 2020a). For application reasons, the tensile properties of ACCs were always in the focus of most of the publications. For example, when aligned native ramie fibers were immersed in $\mathrm{LiCl} / \mathrm{DMAc}$ for $2 \mathrm{~h}$, exceptional longitudinal tensile properties of $460 \mathrm{MPa}$ tensile strength and $28 \mathrm{GPa}$ Young's modulus were obtained (Soykeabkaew et al. 2008). One of the strongest man-made fibers, Bocell, was also used for making ACCs with volume fractions of up to $90 \%$ of fibers, resulting in an average tensile strength of $910 \mathrm{MPa}$ and a Young's modulus of $23 \mathrm{GPa}$ with $8 \%$ elongation at break (Soykeabkaew et al. 2009). The above-mentioned mechanical properties far exceed the values reported for the traditional unidirectional natural fiber reinforced polymer composites. Nevertheless, the majority of the tensile properties of ACCs lies in the interval of 1-20 GPa for Young's modulus and 50-200 MPa for tensile strength (Baghaei and Skrifvars 2020). The results depend on numerous parameters such as the 
origin of the reinforcing fibers, their concentration and alignment, and processing conditions (type of solvent, dissolution or impregnation time and temperature, drying mode, etc.) (Baghaei and Skrifvars 2020). It should be noted that the values obtained with tensile testing also depend on sample geometry and the method used to obtain the specimen elongation (directly from the tensile machine, from extensometer in which gauges are "clipped" on the material, using video extensometers or digital image correlation (DIC)). We will not consider here "contact" extensometer as it may induce damage or additional stress on the sample. Mechanical analysis will be performed using DIC technique; for example, it is commonly used for the analysis of the deformation mechanisms of polymers (Hild and Roux 2006). This method is accurate and provides crucial information on the strain field at macroscale level. It also allows measuring nonuniform deformation and 3D effects. However, DIC is rarely used for natural fiber reinforced polymer composites (Xu et al. 2019; Ramakrishnan et al. 2020) and, to the best of our knowledge, was never considered for the evaluation of the tensile properties of all-cellulose composites.

There are different ways to assess the tensile properties of a material. Below we recall the background equations determining nominal stress $\left(\sigma_{\mathrm{n}}\right)$ and strain $\left(\varepsilon_{\mathrm{n}}\right)$ :

$\sigma_{n}=\frac{F}{A_{0}}$

$\varepsilon_{n}=\frac{d}{L_{0}}=\frac{L-L_{0}}{L_{0}}$

where $\mathrm{F}$ is force, $\mathrm{A}_{0}$ is cross-section area of the sample, $d$ is displacement, and $\mathrm{L}_{0}$ and $\mathrm{L}$ are the initial length of the sample and the length at a given applied force, respectively. The displacement $\mathrm{d}$ can be monitored in different ways. One is using data obtained from the machine displacement sensor; in this work such method will be named "machine approach". The other uses local measurement of sample displacement, i.e. either with video extensometer or DIC techniques; it will be named "local approach".

Irrespective of the approach used, stress-strain dependences are built to characterize the material with Young's modulus in the linear region, maximal stress (often called "tensile strength" or "ultimate stress"), elongation at break and elongation at maximal stress.
The as-obtained results will include different levels of errors depending on the approach used. For instance, the measurement of displacement made with the machine approach includes not only the elongation of the sample in the gauge length region, but also the displacement induced by the stiffness of the tensile machine, the last one inducing erroneous mechanical properties (G'Sell et al. 1992). To exclude this problem which is common for all tensile testing machines, non-contact measurements of the displacement were developed. For example, video extensometer was introduced in the pioneering work of G'Sell et al. (G'Sell et al. 1992) to study highly deforming materials showing "necking", the latter resulting in the alteration of the cross-section during experiment. However, the displacement of the specimen may not be homogeneous along the specimen length. The displacement measured using video extensometer may depend on the location of the marks on the sample and also on the length $L$ which can be either sample length (Eq. 2) or the distance between selected two points. Finally, the most precise (but the most time-consuming) way of having a complete information on the deforming material is digital image correlation that allows obtaining a map of local strains on the specimen surface due to video cameras in stereovision which monitor the displacements of the markers (or speckles) randomly placed on the sample surface (Sutton et al. 2009; Candau et al. 2016).

As far as all-cellulose composites are concerned, the majority of tensile properties were obtained using a machine method (see, for example, ref. Soykeabkaew et al. 2008; Soykeabkaew et al. 2009; Piltonen et al. 2016; Sirviö et al. 2017; Korhonen et al. 2019; Wei et al. 2020; Chen et al. 2020a). Few works report the results obtained with a video extensometer (Kröling et al. 2018; Mat Salleh et al. 2017; Duchemin et al. 2009). In all examples mentioned above nominal stress-strain dependences were used to calculate Young's modulus, tensile strength and elongation at break. The influence of the method on the stress-strain curves and on the values of Young's modulus, tensile strength and elongation at break of all-cellulose composites has never been studied before.

The goal of this work was to analyse the tensile properties of all-cellulose composites applying local and machine approaches and understand the influence of each method on the values of the main mechanical characteristics of the material. Filter paper-based 
ACCs were produced using the ionic liquid 1-ethyl-3methylimidazolium acetate ([EMIM][OAc]) as cellulose solvent, and the influence of the dissolution time on composite morphology, density, crystallinity and optical properties was investigated. Then, the tensile properties of ACCs were evaluated using data obtained directly from the machine and with a local technique, the latter using a two-cameras system and digital image correlation. Stress-strain data corresponding to various approaches were obtained and discussed together with the main tensile characteristics of the composite materials.

\section{Experimental part}

\section{Materials}

Qualitative VWR® Grade 415 filter paper made of pure cellulose was purchased from VWR International, France. Ionic Liquid 1-ethyl-3-methylimidazolium acetate ([EMIM][OAc], purity $>95 \%$ ) was purchased from IoLiTec. The initial moisture content is $0.27 \mathrm{wt} \%$, determined by Karl-Fischer titration. The IL was used as received. Water used in this study was deionized.

\section{Methods}

\section{Manufacturing paper-based ACCs}

The paper-based ACCs manufacturing method is illustrated in Fig. 1. Two pieces of filter papers were dipped in [EMIM][OAc] for $1 \mathrm{~min}$, stacked together and hot pressed at $60{ }^{\circ} \mathrm{C}$. The pressing times were $5 \mathrm{~min}, 10 \mathrm{~min}, 30 \mathrm{~min}$ and $60 \mathrm{~min}$ with the pressure kept constant (around 100 bar). Then the sample was immersed in a large amount of deionized water for about $72 \mathrm{~h}$ (exchanging to fresh water every day) to remove the ionic liquid from the system. Finally, the specimen was dried by hot pressing (around 50 bar, $80{ }^{\circ} \mathrm{C}$ for $2.5 \mathrm{~h}$ and then at $60{ }^{\circ} \mathrm{C}$ for $0.5 \mathrm{~h}$ ). A temperature gradient during drying was shown to prevent the warping and/or heterogeneous contraction of the sample (Chen et al. 2020a). ACCs produced with $5 \mathrm{~min}, 10 \mathrm{~min}, 30 \mathrm{~min}$ and $60 \mathrm{~min}$ impregnation are named P5, P10, P30 and P60, respectively.
Scanning electron microscopy (SEM)

The surface and cross-section morphologies of the filter paper and produced ACCs were observed using a scanning electron microscope (Zeiss Sigma VP FESEM) at an accelerating voltage of $4 \mathrm{kV}$. Prior to examination, the surface and cross section of the samples were coated with a thin layer of gold.

\section{Sample thickness and density}

The density of the filter paper and of the produced ACCs was determined by measuring sample weight and volume. The volume was calculated from the dimensions of the sample measured by using $\mathrm{L} \& \mathrm{~W}$ Micrometer (Lorentzen \& Wettre Products, ABB, Switzerland) with an error of $\pm 1 \mu \mathrm{m}$ or $0.1 \%$ of reading whichever is greater. Porosity was estimated from the density of filter paper and ACCs by using a value of $1.47 \mathrm{~g} \mathrm{~cm}^{-3}$ as the density of cellulose (Sun 2008), using the following equation:

Porosity $(\%)=\left[1-\frac{\rho_{\text {ACCs }}}{\rho_{\text {cellulose }}}\right] \times 100 \%$

\section{Ultraviolet-visible light (UV-Vis) spectroscopy}

The transmittance and haze of the ACC in the visible light region were obtained using a Shimadzu UV-2600 with an ISR-2600 Plus Integrating Sphere Attachment (Shimadzu, Japan). Optical haze was used to quantify the percentage of the forward light scattering as follows:

Haze $=\left[\frac{T_{4}}{T_{2}}-\frac{T_{3}}{T_{1}}\right] \times 100 \%$

where $T_{1}, T_{2}, T_{3}$ and $T_{4}$ are defined as background checking, total transmitted illumination, beam checking and pure diffusive transmittance, respectively.

\section{$X$-ray diffraction $(X R D)$}

XRD data were collected in the reflection mode of an X-ray instrument, SmartLab (RIGAKU), operated at $45 \mathrm{kV}$ and $200 \mathrm{~mA}$. The sample was placed on a sample holder horizontally to incident $\mathrm{X}$-ray and the scans were performed in the $2 \theta$ range from $5^{\circ}$ to $60^{\circ}$ by $\theta / 2 \theta$ mode. Background intensity profile without 


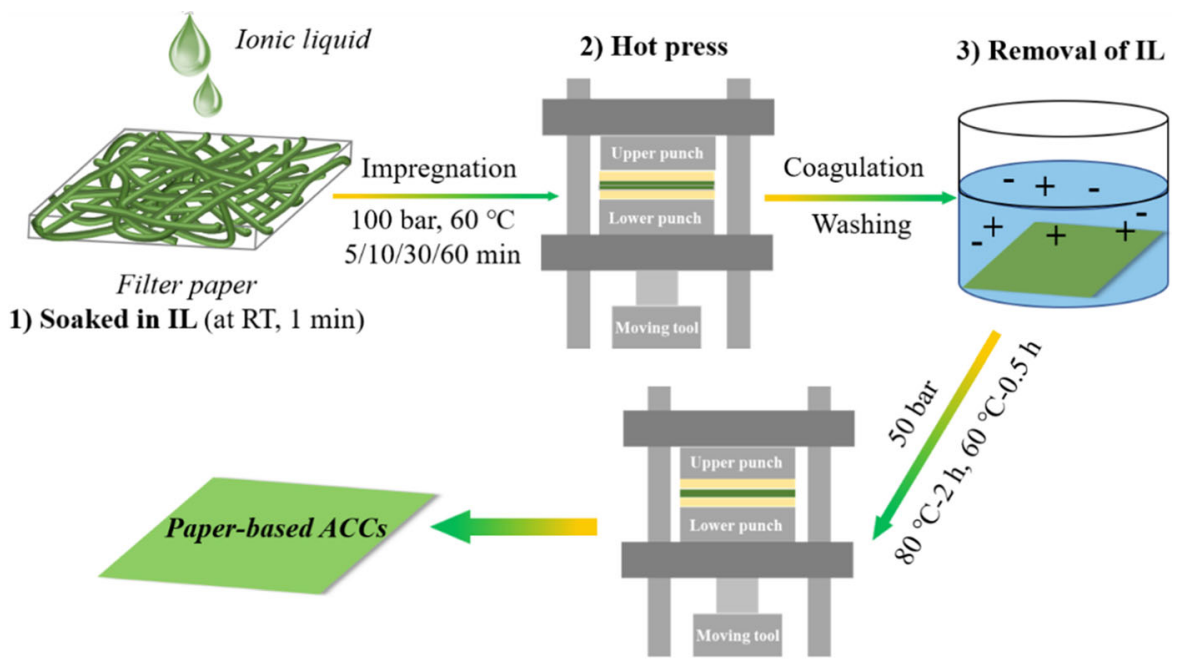

4) Hot press drying

Fig. 1 Illustration of the fabrication of the paper-based ACCs

sample was collected in the same conditions, and it was subtracted from the obtained data. The subtracted data were corrected for the Lorentz-polarization factor for each data point. The background intensity $\left(\mathrm{S}_{\mathrm{bkg}}\right)$ from the amorphous phase of cellulose was estimated by robust smoothing procedure as described elsewhere. The total crystallinity index (CRI) of the initial filter paper and of ACCs was estimated using the ratio of the area of total intensity $\left(S_{\text {total }}\right)$ to that of background intensity $S_{\mathrm{bkg}}$ in the $2 \theta$ range from $10^{\circ}$ to $32^{\circ}$ :

$C R I, \%=100 \% \times\left(1-S_{b k g} / S_{\text {total }}\right)$

The background-corrected profile was fitted with 4 pseudo-Voigt function for cellulose I ((1-10), (110), $(102) /(012)$ and (200) lattice planes) and 3 pseudoVoigt functions for cellulose II ((1-10), (110) and (020) lattice planes). For each polymorph, the ratio of amplitude for the lattice planes were fixed to be the ratio of the square of structure factor (Nishiyama et al. 2002; Langan et al. 2001). The deviation of diffraction positions was limited to be close to those calculated from the unit cell parameters of the crystal structure of cellulose I and II. The software LMFIT (Newville et al. 2016) was used for the fitting. As the mass absorption coefficients are identical for crystalline polymorphs, quantitative phase analysis was performed using the single peaks of cellulose polymorphs (Alexander and Klug 1984). The intensities for the (1-
10) lattice plane of cellulose I $\left(I_{1-10(1)}\right)$ and cellulose II $\left(I_{1-10(2)}\right)$ can be expressed as a function of the structure factor $F_{1-10(\alpha)}$, the volume of unit cell $V_{(\alpha)}$ and the volume fraction $v_{\alpha}$ of each crystalline phase ( $\alpha$ being either cellulose I or II crystal) in the total cellulose crystal, as follows:

$I_{1-10(\alpha)}=\frac{K}{V_{(\alpha)}^{2}}\left|F_{1-10(\alpha)}\right|^{2} v_{\alpha}$

where $\mathrm{K}$ is a constant for the instrument and sample.

Thus, the ratio of the volume fraction of cellulose II to cellulose $\mathrm{I}, v_{2} / v_{1}$, is as follows:

$\frac{v_{2}}{v_{1}}=\frac{2.27 I_{1-10(2)}}{I_{1-10(1)}}$

where the coefficient 2.27 was calculated from the unit cell volume and the structure factor for the (1-10) lattice of cellulose I (Nishiyama et al. 2002) and cellulose II (Langan et al. 2001). This equation was used to calculate the volume fraction of cellulose II over the total crystalline cellulose as follows:

Cell $_{I I}$ vol $\%=\frac{v_{2}}{v_{1}+v_{2}}$

\section{Tensile testing}

The mechanical properties of the filter paper and ACCs were studied using the electro-mechanical 
tensile test machine Zwick- $2.5 \mathrm{kN}$ at $23{ }^{\circ} \mathrm{C}$ and $50 \%$ relative humidity. Figure 2a presents the geometry of the tensile sample with a gauge length $\left(\mathrm{L}_{0}\right)$ and a width (W) of $15 \mathrm{~mm}$ and $10 \mathrm{~mm}$, respectively. Sample thickness was measured at different locations within the gauge length area for each sample with highprecision caliper and the maximal standard deviation was $\pm 0.005 \mathrm{~mm} / 50 \mathrm{~mm}$. The tensile measurements were carried out at a strain rate of $1 \times 10^{-3} \mathrm{~s}^{-1}$.

The first set of force-displacement data was collected directly from the tensile machine (machine approach). In parallel, two-cameras stereovision system was used to monitor the local displacements of ACC (Fig. 2b). Stereovision provides a 3D image of sample surface using a superposition of two 2D plane images, and DIC allows building a 2D displacement field on sample surface. In this work, a 3D DIC method was used. The advantages of 3D DIC method compared to the 2D DIC can be found elsewhere (Sutton et al. 2008). The calibration of 3D DIC was done until a projection error of 0.05 was reached (Sutton et al. 2009).

To monitor the local displacements, the front surface of the sample was coated with a thin layer of white paint on which black ink was sprayed to generate a random speckle pattern (Fig. 2c). The displacement field was obtained by tracking the positions of speckle patterns on a specimen surface before and after the deformation of the body. Practically, two sets of images - corresponding to two different mechanical states (i.e. reference and deformed) - are recorded by two fixed cameras set
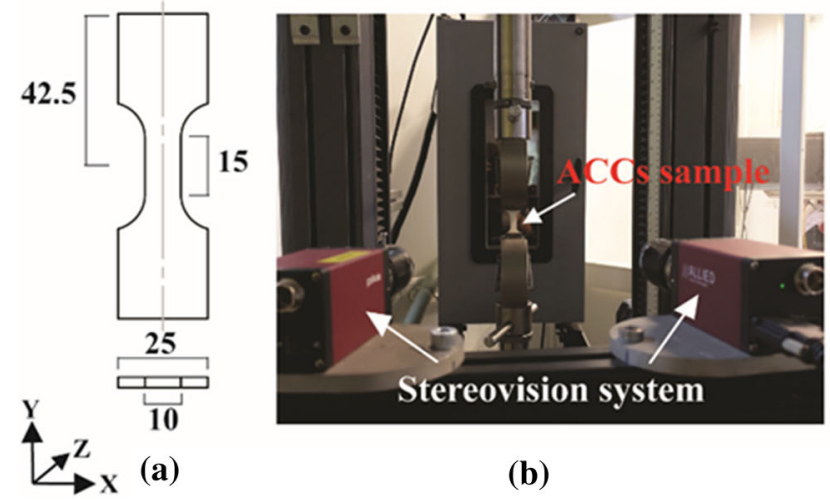

(b)

Fig. 2 The tensile testing setup used in this study: a geometry of a tensile sample, values are in $\mathrm{mm}$; b experimental setup with stereovision system; c speckled sample for the DIC analysis and d example of true strain field obtained by DIC analysis using with in-between fixed angle. As grey level cannot be a unique characteristic of a "point" on sample surface, neighboring pixels must be used to build a displacement field using the initial and deformed pictures. Such a collection of pixel values is called a subset. Large speckles (e.g. more than 20 pixels) were avoided as they need large subset sizes which reduces the spatial resolution of the analysed zone.

The displacement fields were built from images recorded by a pair of cameras AVT PIKE 5 Megapixels with a $2 / 3^{\prime} \mathrm{CCD}$ sensors and $50 \mathrm{~mm}$ Schneider Kreuzwach objectives. The images were taken every second and post-processed using VIC-3D software (S. Vic-3D 2007) (Fig. 2d). The first step of the analysis consists of creating a regular mesh in the region of interest. The meshing allows tracking the speckle pattern during the tensile test. In this study, the subset was 23 pixels with a step of 5 and filter size of 13 . More details on the selection of parameters and analysis of data are given in the Supporting Information.

As mentioned in the Introduction, there are different ways of using digital image correlation approach. One option is to use it as a video extensometer: we select two different locations of "virtual extensometers", one monitoring strain evolution along $\mathrm{E}_{\mathrm{a}}$ and the other along $E_{b}$ (Fig. 2d). Different locations of the virtual extensometers are selected to better understand the influence of their location on strain distribution over the sample and the corresponding stress-strain dependences. Another option is to average the displacement over a given surface; here we will use

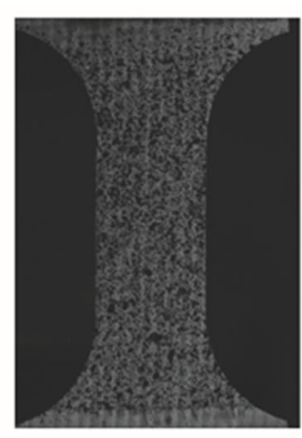

(c)

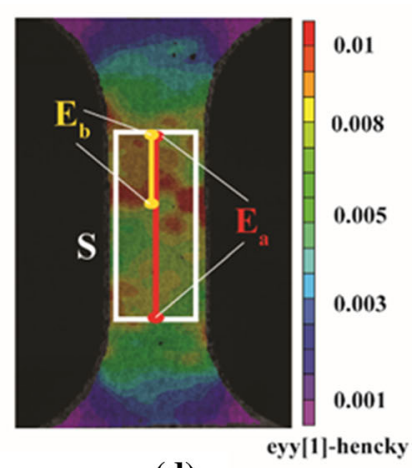

(d)
VIC-3D software, $E_{a}$ and $E_{b}$ are locations of a virtual extensometer and $\mathrm{S}$ is the surface of the rectangle over which the true strain in the longitudinal direction is averaged 
surface $\mathrm{S}$ shown by a rectangle in Fig. 2 d. While virtual extensometer provides nominal strain evolution over time and further nominal stress-strain response (Eqs. 1 and 2), the evolution of true strain over time is obtained from VIC-3D software by averaging true strain distribution over the surface $S$. We recall below the relationship between true strain $\varepsilon_{\text {true }}$ and nominal strain $\varepsilon_{n}$ :

$\varepsilon_{\text {true }}=\ln \left(1+\frac{L-L_{0}}{L_{0}}\right)=\ln \left(1+\varepsilon_{n}\right)$

true stress $\sigma_{\text {true }}$ is then determined as follows:

$\sigma_{\text {true }}=\frac{F}{A(t)}=\frac{F}{A_{0} \exp ^{\left(-\varepsilon_{\text {true }}\right)}}$

where $A(t)$ is sample cross-section area at a given moment.

\section{Results and discussions}

To better understand the mechanical performance of ACCs and the influence of the analysis method (machine vs local), material properties such as density, morphology and crystallinity must be first examined. Therefore, we start with the analysis of the evolution of ACC characteristics as a function of processing conditions. Then, the local approach using different DIC analyses vs machine approach applied to ACC will be addressed, using one sample as an example. This study will be used to setup the methodology of tensile tests analysis. Finally, the tensile values (tensile (maximal) strength, strain at maximal strength, Young's modulus and work of fracture) obtained via machine data and local approach using stereovision system are compared and discussed.

\section{Density and crystallinity of ACCs}

The evolution of ACC density and porosity as a function of impregnation time is shown in Fig. 3. Density increases with the impregnation time under pressure indicating a strong decrease in material porosity, from $73 \%$ for filter paper and $42 \%$ for P5 to $11 \%$ for P60 (Fig. 3). No noticeable evolution is recorded after $30 \mathrm{~min}$ of dissolution.

The ACCs XRD profiles, crystallinity and cellulose allomorphs as a function of impregnation time are

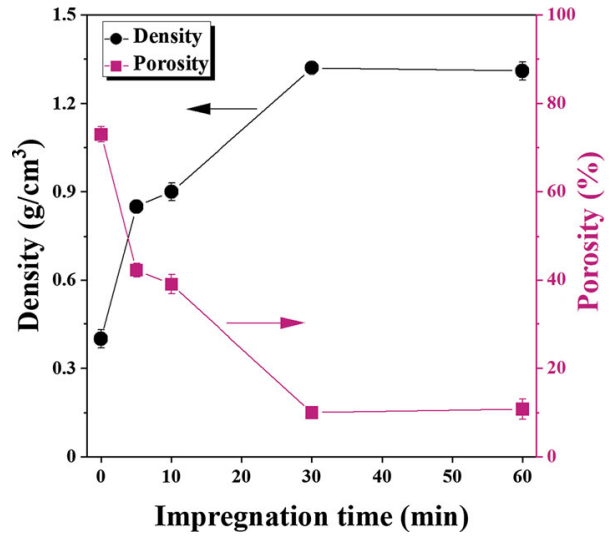

Fig. 3 Density and porosity of ACC as a function of impregnation time. The properties of filter paper are shown at $t=0$. The lines are given to guide the eye

shown in Fig. 4. The filter paper was theoretically composed of native cellulose which is the cellulose I allomorph. However, a small diffraction peak at $2 \theta \approx 12^{\circ}$, which indicates cellulose II allomorph, was also observed (Fig. 4a). It has been reported that a paper sheet, despite supposed to be native cellulose, may contain a small amount of cellulose II crystalline structure (Sirviö et al. 2017; Piltonen et al. 2016). The amount of cellulose II strongly increases after $5 \mathrm{~min}$ impregnation, from around 7 to $34 \mathrm{vol} \%$, but then does not vary significantly (Fig. 4b). Crystallinity drops from around $56 \%$ to $43 \%$ for 5 min impregnation and then slowly decreased to around $40 \%$ for longer impregnation times. Based on these results we suppose that dissolution was almost stopped after the first $5 \mathrm{~min}$. The reason is that the total cellulose concentration was too high ( $>40 \mathrm{wt} \%)$, above the dissolution limit in the ionic liquid. It was suggested that at least 3 ionic liquid molecules per anhydroglucose unit are needed to dissolve cellulose in [EMI$\mathrm{M}][\mathrm{OAc}]$ which corresponds to a maximum cellulose solubility of around 25-27 wt\% (Le et al. 2014). It should also be taken into account that the viscosity of the dissolved phase is high and dissolution (impregnation) was performed in static conditions, without mixing, also preventing cellulose dissolution.

\section{Morphology and optical properties of the ACCs}

To provide a direct evidence of the gradient dissolution of the fibers in the filter paper induced by the impregnation time and compression, the surface and 


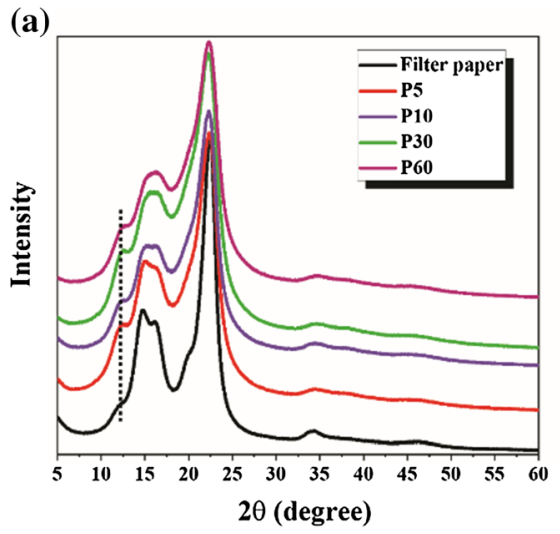

Fig. 4 a X-ray diffractograms of filter paper and the produced ACCs, the black dotted line indicates the characteristic peak of cellulose II. b The total crystallinity index and cellulose II

cross-section morphologies of the initial paper and of obtained ACCs were examined by SEM (Fig. 5). The starting material shows individual fibers typical for a filter paper with large number of voids, as confirmed by the low density. After 5 min of impregnation, fibers can still be distinguished (Fig. 5, images of the surface on the top row) but they are well embedded into the dissolved cellulose matrix demonstrating excellent adhesion. The cross-section shows some remaining pores (Fig. 5, lower row). After $10 \mathrm{~min}$ and longer impregnation times the fibrous structure of the paper had almost disappeared and no pores can be seen. Figure 5 also shows the decrease of ACCs crosssection. SEM observations are in-line with density and XRD results: cellulose that could be dissolved was dissolved during the first $5 \mathrm{~min}$ (strong increase of cellulose II fraction and drop of crystallinity) and further morphology evolution is due to the distribution

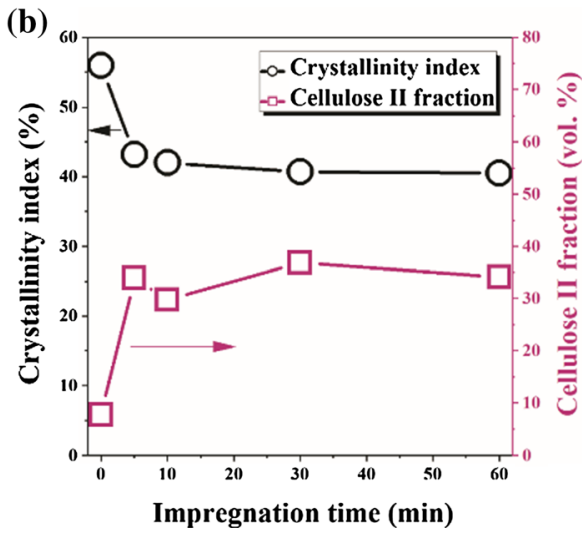

volume fraction of ACC as a function of impregnation time, the lines are given to guide the eye. The properties of filter paper are shown at $\mathrm{t}=0$

of dissolved phase within the pores under pressure (no evolution of cellulose II fraction and crystallinity but continuous increase in density).

The optical properties of ACCs are correlated with their morphology. Figure 6 displays the optical transmittance and haze of the filter paper and the asprepared ACCs. As the filter paper consists of randomly packed irregular cellulose fibers and micron-size voids, it is opaque in the visible light due to massive backward light scattering and possess almost $100 \%$ transmission haze because of strong forward light scattering. In ACCs, the dissolved cellulose fills the voids with the matter of the same refractive index as fibers, surface roughness is decreased and the material becomes transparent in the visible light (Fig. 6a). Morphology homogenization also decreases the forward light scattering resulting in the reduction of haze (Fig. 6b). Both
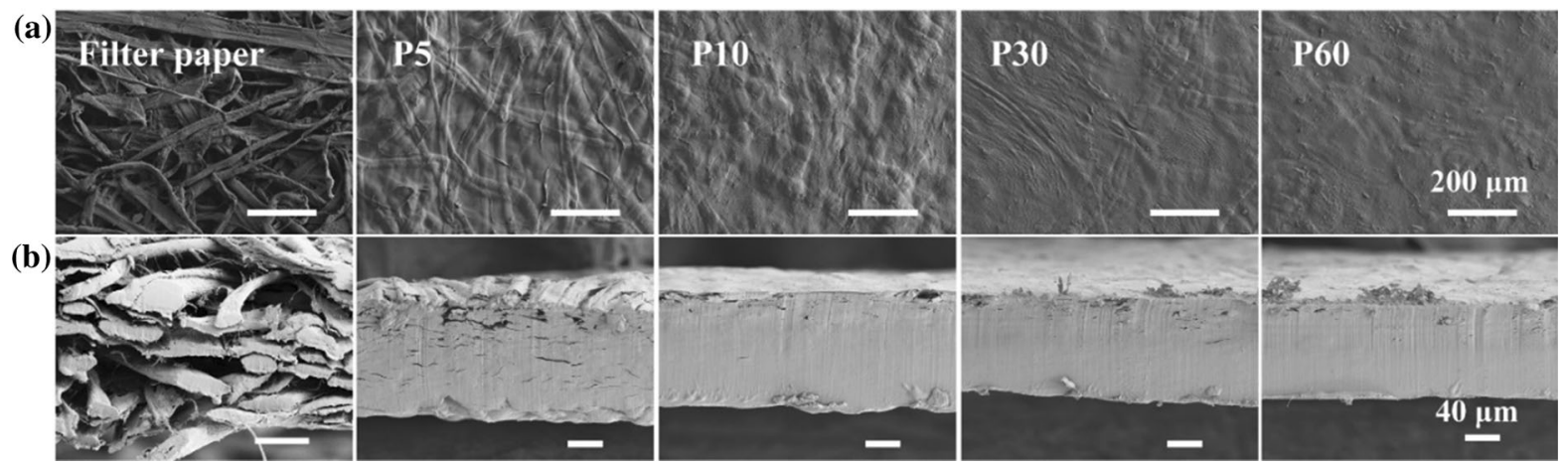

Fig. 5 a Upper set of images: the surface of filter paper and ACCs P5, P10, P30 and P60; b lower set of images: cross-section of filter paper and of the corresponding ACCs. The scale bar for all images on the upper set is $200 \mu \mathrm{m}$ and on the lower set $40 \mu \mathrm{m}$ 
(a)

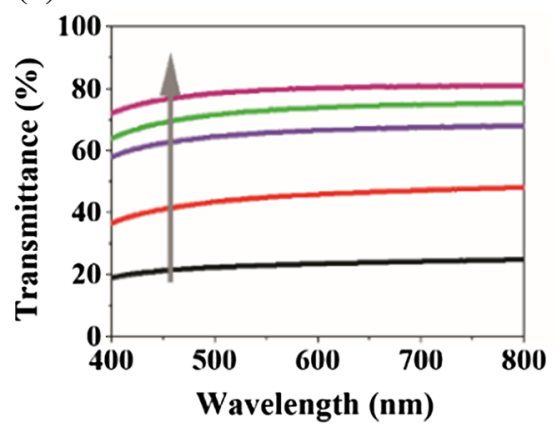

(c)

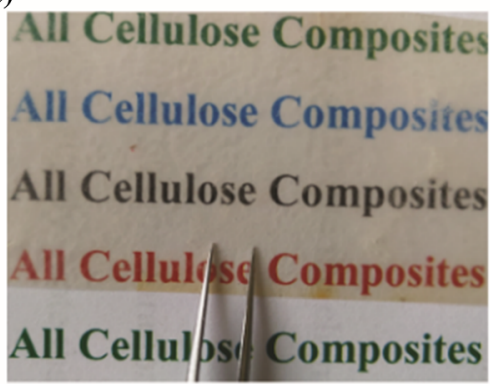

(b)

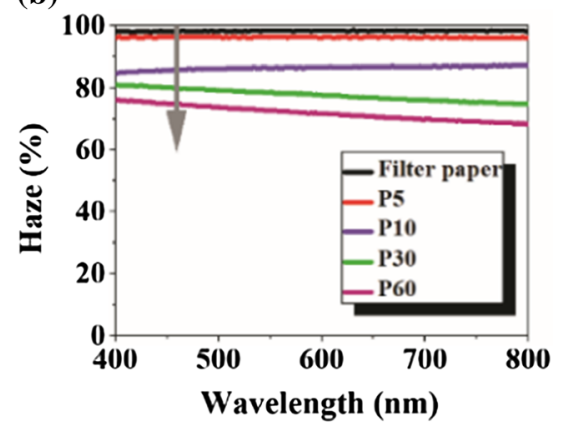

(d)

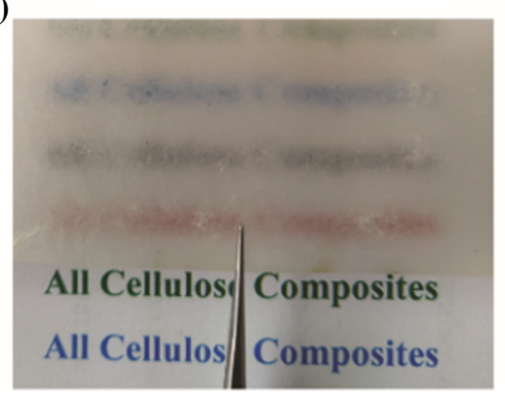

Fig. 6 Optical transmittance (a) and haze (b) of filter paper and the ACCs, ACC P60 in close contact with the colored letters underneath to show its high light transmittance (c) and $1 \mathrm{~cm}$ away from the same substrate (d) to indicate its high haze

transmittance and haze show a sharp transition of properties at impregnation times longer than $5 \mathrm{~min}$, inline with the ACC morphology (Fig. 5) and density (Fig. 4). It is worth noting that ACCs P60 is transparent (optical transmittance $\sim 80 \%$ at $550 \mathrm{~nm}$ ) but still with high haze $(>70 \%$ at $550 \mathrm{~nm})$, which makes it promising as photonic material for optoelectronic applications such as solar cells, as haze increases light scattering and absorption (Hou et al. 2020; Chen et al. 2020b). The digital photographs of ACC P60 confirm high transmittance and high haze (Fig. 6c, d).

Mechanical properties

\section{Local versus machine approach}

Sample P5 was used to test different approaches for the analysis of ACC tensile properties. An example of strain distributions over the sample surface obtained with a local (DIC) approach for tensile loading is shown in Fig. 7. This type of analysis was performed for all other samples. Figure 7 shows that the strain field is heterogeneous with strain concentration on the upper side; failure is initiated in this area.
To check if this heterogeneity induces differences in stress-strain curves, the nominal strain was plotted as a function of time (Fig. 8a) and then converted into nominal stress-strain curves (Fig. 8b) for two locations of the virtual extensometer, $\mathrm{E}_{\mathrm{a}}$ and $\mathrm{E}_{\mathrm{b}}$ (see details Methods section). Figure 8 a shows similar curves for the two positions until a strain of 0.03. Some differences are then observed due to the strong localisation of the deformation and the specimen failure. It is translated into a quite minor difference in the strain at failure, 0.06 (for $\mathrm{E}_{\mathrm{b}}$ ) versus 0.05 (for $\mathrm{E}_{\mathrm{a}}$ ). The strain localisation is well captured by $\mathrm{E}_{\mathrm{b}}$ while $\mathrm{E}_{\mathrm{a}}$, characterized by a higher gauge length $\mathrm{L}_{0}$, is less sensitive to this strain localisation as it represents the average of the nominal strain distribution over the corresponding gauge length. As stress-strain curves are practically identical, Young's modulus and tensile strength are the same for both locations of the virtual extensometer. However, this may not always be true especially for the determination of the strain to failure.

Next, we compare nominal stress-strain curve obtained from the virtual extensometer $\mathrm{E}_{\mathrm{a}}$ with that averaged over the surface $\mathrm{S}$ (Fig. 8c). A minor difference is observed for the strain at failure. Then 

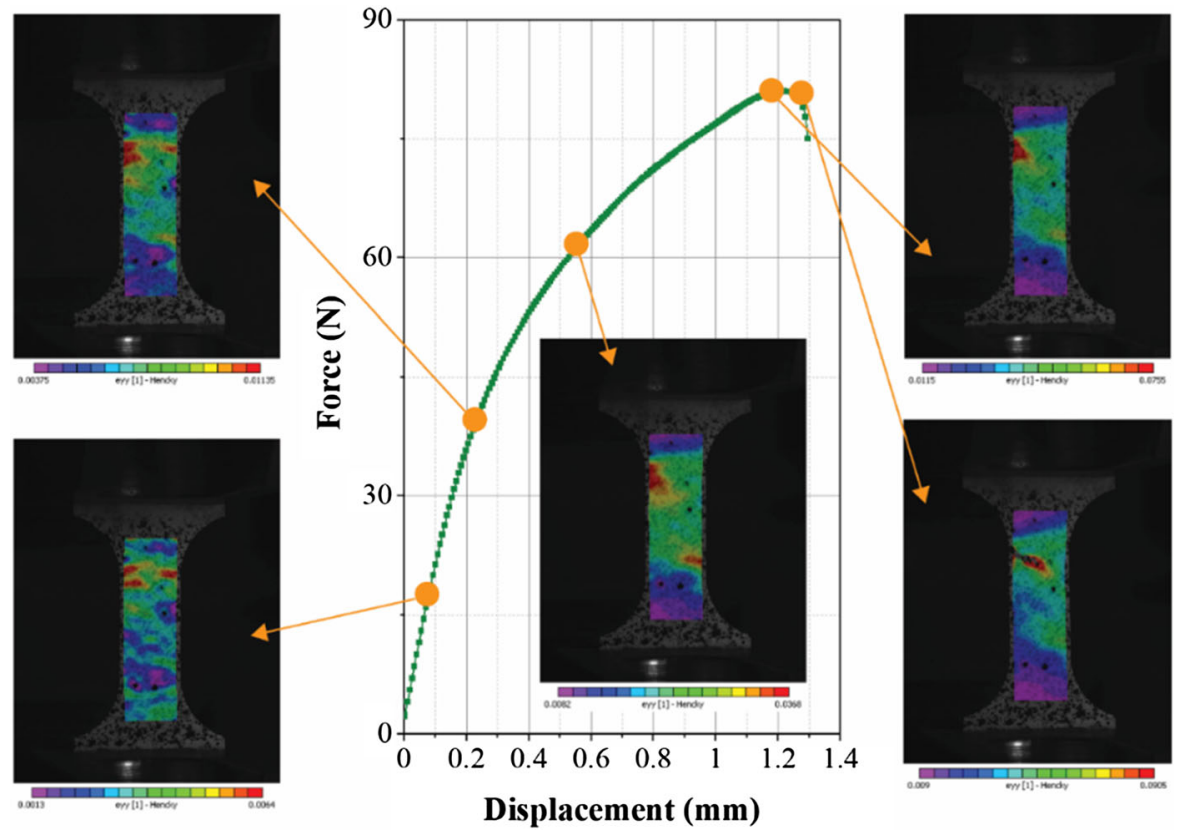

Fig. 7 The true strain field in the longitudinal direction obtained by DIC as function of displacement for the sample P5

(a)

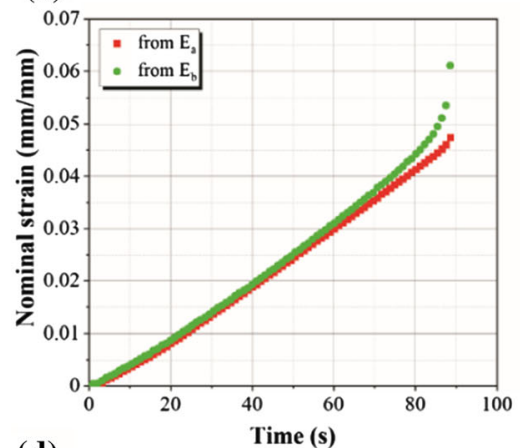

(d)

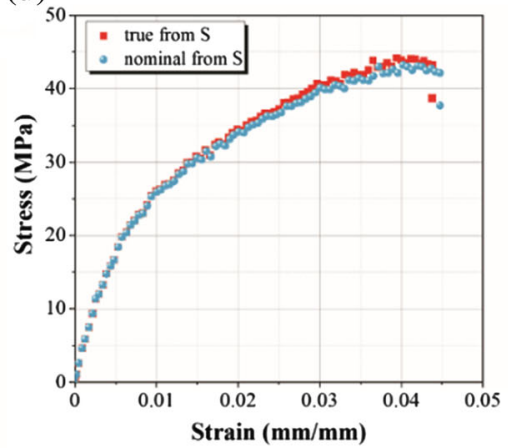

(b)

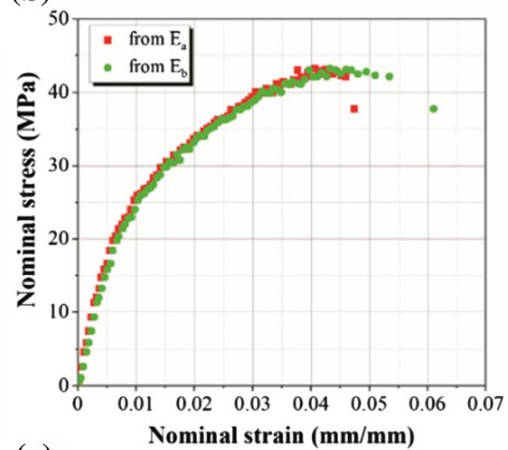

(e)

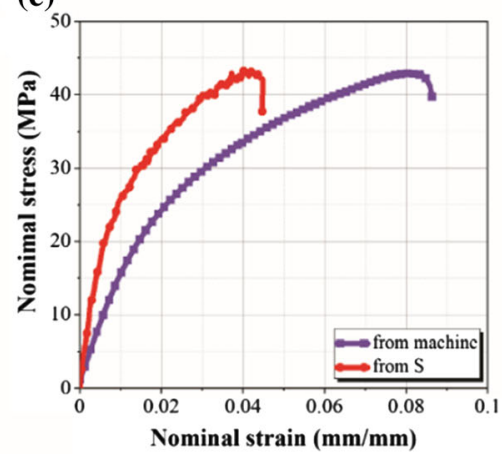

(c)

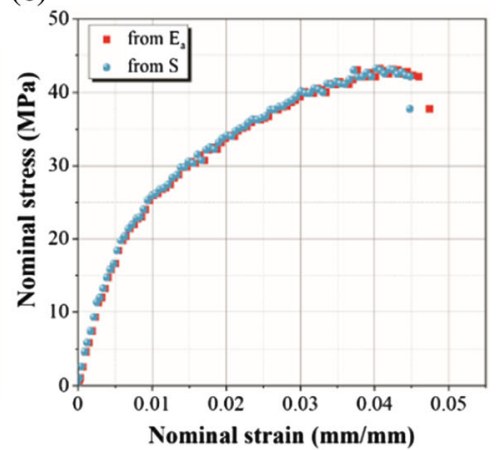

Fig. 8 Comparison of tensile data for P5 obtained with different approaches: a nominal strain as a function of extension time collected from the virtual extensometers $\mathrm{E}_{\mathrm{a}}$ and $\mathrm{E}_{\mathrm{b}}$, $\mathbf{b}$ nominal stress-strain curves from the virtual extensometers $E_{a}$ and $E_{b}$; c nominal stress-strain curves from the virtual extensometer $E_{a}$ and over the surface $\mathbf{S}$; d nominal and true stress-strain curves obtained with stereovision system over the surface $S$ and e nominal stress-strain curves obtained from the machine and with stereovision system over the surface $\mathrm{S}$ 
nominal and true stress-strain curves are compared for the case when the strain is averaged over the surface $S$ (Fig. 8d). The true stress is slightly higher than the nominal one with slightly lower strain at failure. Such coincidence can be explained by the overall low deformation of the sample. For materials exhibiting large deformations more differences between nominal and true strain-stress curves are usually recorded.

Finally, Fig. 8e compares the nominal stress-strain curve directly derived from the machine (machine approach) with that derived from stereovision and averaged over the surface $S$ (local approach). Here the difference is significant: the strain at failure determined with machine approach is twice higher than that determined with DIC. With nominal tensile strength being almost the same, Young's modulus calculated with the local approach will be much higher than that calculated from machine approach. We remind that the latter provides results with the systematic error arising from the equipment input.

\section{Tensile properties of ACCs}

Young's modulus, tensile strength, strain at maximal stress and toughness were calculated from nominal and true stress-strain curves (Figure S1, Supporting Information) derived from the machine and local approaches, the latter using stereovision system and averaged over the surface S. All mechanical characteristics of the filter paper and ACCs are given in Table S1, their dependence on material density is shown in Fig. 9 and on the impregnation time in Figure S2.

The increase in ACC density, which corresponds to the increase of impregnation time, leads to a strong increase of Young's modulus, independent of the method used to determine it (Fig. 9a). All values calculated from nominal stress-strain curves via a local approach using stereovision are much higher than those calculated from nominal ones via machine approach, as expected from Fig. 8e. We also plotted Young's modulus as a function of crystallinity (Figure S3); modulus decrease with crystallinity increase shows that the crystallinity does not play an important role here as it is the presence of pores and heterogeneities that control mechanical properties. It has been demonstrated in the previous studies that density plays the essential role in the mechanical performance of ACCs (Korhonen et al. 2019; Chen et al. 2020a); an adequate comparison with literature can thus be made only for the materials either of the same density or for the specific values of mechanical characteristics. Unfortunately, the density of ACC is not always reported. To the best of our knowledge, for paperbased isotropic all-cellulose composites, only the study from Piltonen et al. (Piltonen et al. 2016) reported density values. The calculated specific strength and modulus values obtained in this work are listed in Table S2 in the Supporting Information and compared with those reported by Piltonen et al. (Piltonen et al. 2016). Considering that the type of approach (machine or contact or non-contact extensometer) is rarely specified, the comparison of moduli values with the results obtained in literature becomes even more delicate.

Similar to the Young's modulus, tensile strength and toughness increase with density increase. Interestingly, the strain at break also increases, from 4 to $9 \%$, the latter corresponding to the ACC with a density of around $0.9 \mathrm{~g} / \mathrm{cm}^{3}$ (impregnation time $10 \mathrm{~min}$ ); at higher density, the strain at break remains on the same level. A certain porosity improves material ductility.

Figures 8 and 9 show that while Young's modulus calculated from nominal stress-strain curves using a local approach is higher than that calculated using the machine approach, the opposite was obtained for the strain at maximum stress and toughness. A lower toughness obtained from local approach is due to much lower nominal strains at maximum stress as compared to those obtained via machine approach. Tensile strength values did not depend on the approach used within the errors.

Overall, a simple strategy of filter paper impregnation with ionic liquid for 30-60 min allowed increasing the tensile strength by almost 10 times, of Young's modulus by 5 times and of toughness by 25 times as compared to the initial filter paper. In order to make an adequate comparison with literature results, the properties of the starting material should be considered. In our case, the nominal tensile strength values calculated from the local approach and from machine approach are similar. Supposing this is the case for ACCs published in other works, the results on various isotropic ACCs can be put together. As presented in Fig. 10, Sirviö et al. (2017) obtained a very high increase of nominal tensile strength of dissolving pulp-based ACCs, up to 24 times, but the absolute value of tensile strength was only around $76 \mathrm{MPa}$, 
(a)

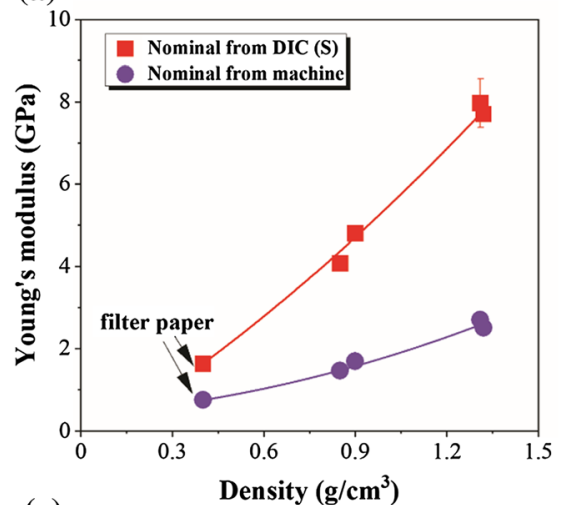

(c)

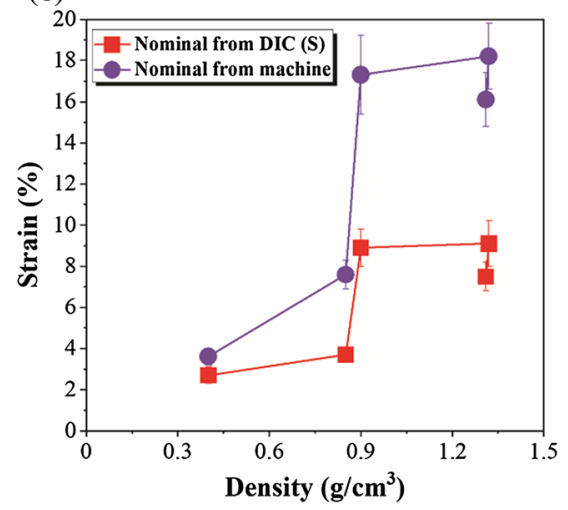

(b)

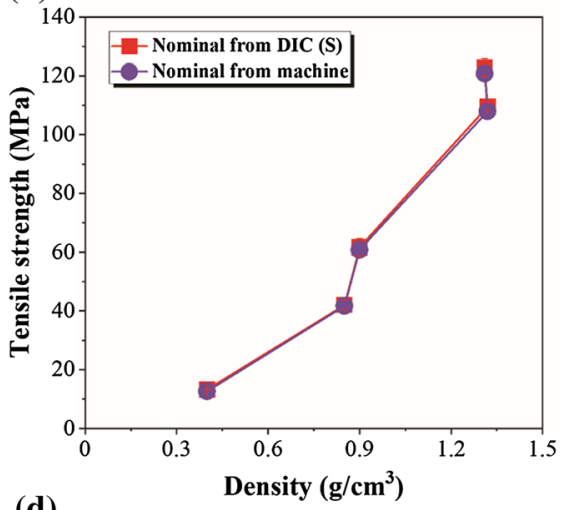

(d)

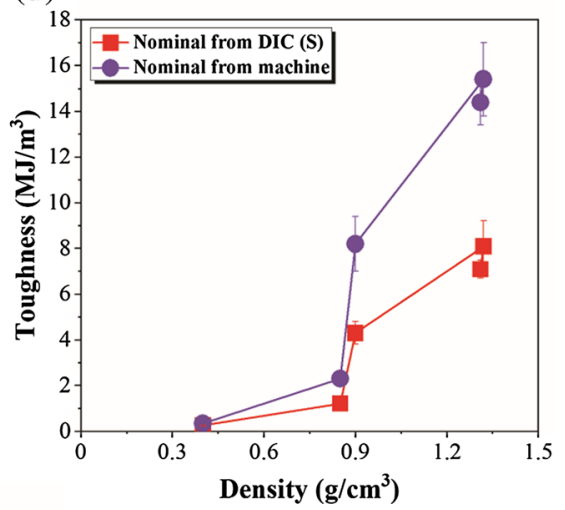

Fig. 9 Young's modulus, tensile strength, strain at maximal stress and toughness of the filter paper and ACCs as a function of density. When errors are not visible, they are within the size of the symbol. The lines are given to guide the eye

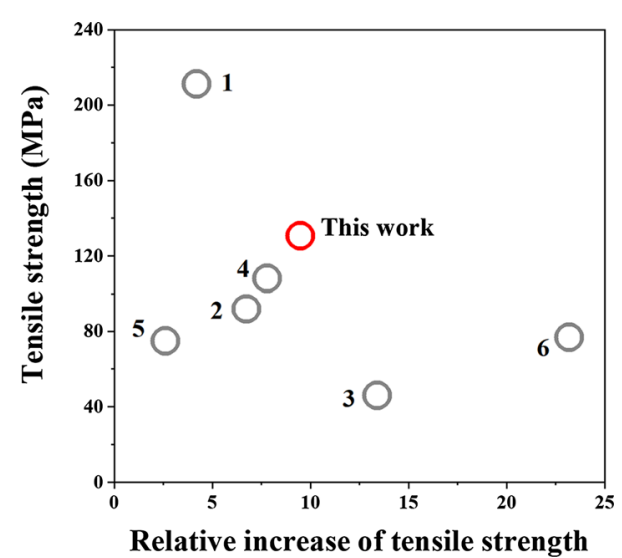

Fig. 10 A comparison of maximal tensile stress and of the increase of maximal tensile stress as compared to the starting filter paper for isotropic paper-based ACCs (1, Nishino and Arimoto 2007; 2, Duchemin et al. 2009; 3, Piltonen et al. 2016; 4, Wei et al. 2020; 5, Han and Yan 2010; 6, Sirviö et al. 2017)

most probably because of the low mechanical properties of the starting material. Nishino and Arimoto
(Nishino and Arimoto 2007) reported a filter paperbased ACC with a nominal highest tensile strength of $211 \mathrm{MPa}$, but the initial filter paper was much stronger, with tensile strength around $50 \mathrm{MPa}$. In the current study the tensile strength of the initial filter paper was around $13 \mathrm{MPa}$. The comparison of the best values of tensile strength vs its increase as compared to the strength of the initial paper is shown in Fig. 10.

\section{Conclusions}

All-cellulose composites were made via controlled impregnation with ionic liquid, [EMIM][OAc]. First, the properties of the composites (density, porosity, crystallinity, cellulose II fraction, transmittance, haze and morphology) were investigated as a function of impregnation time. The results showed that cellulose was dissolved during the first $5 \mathrm{~min}$, and further 
evolution of properties was due to the distribution of dissolved phase within the pores.

Tensile testing was conducted using different methods to obtain the specimen elongation: i) data directly taken from machine sensors and ii) using digital image correlation technique and local approach. The latter excludes the errors related to the stiffness of the tensile testing machine itself. Although the nominal tensile strength values from DIC and machine are similar, the nominal Young's modulus, strain at maximal stress and, as a consequence, toughness, are very different. This must be taken into account when comparing results from different literature sources. ACC fabrication strategy using in this work resulted in the increase of tensile strength, Young's modulus and toughness by almost 10, 5 and 25 times, respectively, as compared to the initial filter paper.

Acknowledgments The financial support from Business Finland (Grant No. 211599), FinnCERES funding, Foundation for Aalto University Science and Technology, Stora Enso Oyj and UPM-Kymmene Oyj is gratefully acknowledged. Authors wish to thank Separation Research Oy Ab and Fibertus Oy for collaboration. We also acknowledge the provision of facilities and technical support by Aalto University at OtaNanoNanomicroscopy Center (Aalto-NMC).

Funding Open access funding provided by Aalto University. Business Finland (Grant No. 211599), FinnCERES funding, Foundation for Aalto University Science and Technology, Stora Enso Oyj and UPM-Kymmene Oyj.

Data availability Data can be provided upon request.

Code availability Not applicable.

\section{Declaration}

Conflict of interest The authors declare that they have no conflict of interest.

Open Access This article is licensed under a Creative Commons Attribution 4.0 International License, which permits use, sharing, adaptation, distribution and reproduction in any medium or format, as long as you give appropriate credit to the original author(s) and the source, provide a link to the Creative Commons licence, and indicate if changes were made. The images or other third party material in this article are included in the article's Creative Commons licence, unless indicated otherwise in a credit line to the material. If material is not included in the article's Creative Commons licence and your intended use is not permitted by statutory regulation or exceeds the permitted use, you will need to obtain permission directly from the copyright holder. To view a copy of this licence, visit http://creativecommons.org/licenses/by/4.0/.

\section{References}

Alexander L, Klug HP (1948) Basic aspects of X-ray absorption in quantitative diffraction analysis of powder mixtures. Anal Chem 20:886-889

Baghaei B, Skrifvars M (2020) All-Cellulose composites: a review of recent studies on structure. Proper Appl Mol 25:2836

Candau N, Pradille C, Bouvard JL, Billon N (2016) On the use of a four-cameras stereovision system to characterize large 3D deformation in elastomers. Polym Test 56:314-320

Capiati NJ, Porter RS (1975) The concept of one polymer composites modelled with high density polyethylene. J Mater Sci 10:1671-1677

Chen F, Sawada D, Hummel M, Sixta H, Budtova T (2020a) Unidirectional all-cellulose composites from flax via controlled impregnation with ionic liquid. Polymers 12:1010

Chen F, Xiang W, Sawada D, Bai L, Hummel M, Sixta H, Budtova T (2020b) Exploring large ductility in cellulose nanopaper combining high toughness and strength. ACS Nano 14:11150-11159

Duchemin BJ, Mathew AP, Oksman K (2009) All-cellulose composites by partial dissolution in the ionic liquid 1-butyl-3-methylimidazolium chloride. Compos A Appl S 40:2031-2037

G'Sell C, Hiver JM, Dahoun A, Souahi A (1992) Video-controlled tensile testing of polymers and metals beyond the necking point. J Mater Sci 27:5031-5039

Han D, Yan L (2010) Preparation of all-cellulose composite by selective dissolving of cellulose surface in $\mathrm{PEG} / \mathrm{NaOH}$ aqueous solution. Carbohydr Polym 79:614-619

Hild F, Roux S (2006) Digital image correlation: from displacement measurement to identification of elastic properties-a review. Strain 42(2):69-80

Hou G, Liu Y, Zhang D, Li G, Xie H, Fang Z (2020) Approaching theoretical haze of highly transparent allcellulose composite film. ACS Appl Mater Interfaces 12:31998-32005

Huber T, Bickerton S, Mussig J, Pang S, Staiger MP (2012) Solvent infusion processing of all-cellulose composite materials. Carbohydr Polym 90:730-733

Khakalo A, Tanaka A, Korpela A, Hauru LKJ, Orelma H (2019) All-wood composite material by partial fiber surface dissolution with an ionic liquid. ACS Sustain Chem Eng 7:3195-3202

Korhonen O, Sawada D, Budtova T (2019) All-cellulose composites via short-fiber dispersion approach using $\mathrm{NaOH}-$ water solvent. Cellulose 26:4881-4893

Kröling H, Duchemin B, Dormanns J, Schabel S, Staiger MP (2018) Mechanical anisotropy of paper-based all-cellulose composites. Compos A Appl S 113:150-157

Labidi K, Korhonen O, Zrida M, Hamzaoui AH, Budtova T (2019) All-cellulose composites from alfa and wood fibers. Ind Crop Prod 127:135-141 
Langan P, Nishiyama Y, Chanzy H (2001) X-ray structure of mercerized cellulose II at $1 \AA$ resolution. Biomacromol 2:410-416

Le KA, Rudaz C, Budtova T (2014) Phase diagram, solubility limit and hydrodynamic properties of cellulose in binary solvents with ionic liquid. Carbohydr Polym 105:237-243

Mat Salleh M, Magniez K, Pang S, Dormanns JW, Staiger MP (2017) Parametric optimization of the processing of allcellulose composite laminae. Adv Manuf Polym Compos Sci 3:73-79

Newville M, Stensitzki T, Allen DB (2016) LMFIT: non-linear least-square minimization and curve-fitting for python. Astrophysics Source Code Library, Michigan Technological University, Houghton

Nishino T, Arimoto N (2007) All-cellulose composite prepared by selective dissolving of fiber surface. Biomacromol 8:2712-2716

Nishino T, Matsuda I, Hirao K (2004) All-cellulose composite. Macromolecules 37:7683-7687

Nishiyama Y, Langan P, Chanzy H (2002) Crystal structure and hydrogen-bonding system in cellulose $\mathrm{I} \beta$ from synchrotron $\mathrm{X}$-ray and neutron fiber diffraction. J Am Chem Soc 124:9074-9082

Piltonen P, Hildebrandt NC, Westerlind B, Valkama JP, Tervahartiala T, Illikainen M (2016) Green and efficient method for preparing all-cellulose composites with $\mathrm{NaOH} /$ urea solvent. Compos Sci Technol 135:153-158

Ramakrishnan KR, Corn S, Le Moigne N, Ienny P, Slangen P (2020) Experimental assessment of low velocity impact damage in flax fabrics reinforced biocomposites by coupled high-speed imaging and DIC analysis. Compos A Appl S 1:106137

S. Vic-3D, ®Software (2007) Correlated solutions incorporated, Columbia. https://www.correlatedsolutions.com/vic-3d/

Sirviö JA, Visanko M, Hildebrandt NC (2017) Rapid preparation of all-cellulose composites by solvent welding based on the use of aqueous solvent. Eur Polym J 97:292-298
Soykeabkaew N, Arimoto N, Nishino T, Peijs T (2008) Allcellulose composites by surface selective dissolution of aligned ligno-cellulosic fibres. Compos Sci Technol 68:2201-2207

Soykeabkaew N, Nishino T, Peijs T (2009) All-cellulose composites of regenerated cellulose fibres by surface selective dissolution. Compos A Appl S 40:321-328

Spörl JM, Batti F, Vocht MP, Raab R, Müller A, Hermanutz F, Buchmeiser MR (2017) Ionic liquid approach toward manufacture and full recycling of all-cellulose composites. Macromol Mater Eng 303:1700335

Sun CC (2008) Mechanism of moisture induced variations in true density and compaction properties of microcrystalline cellulose. Int J Pharm 346:93-101

Sutton MA, Yan JH, Tiwari V, Schreier HW, Orteu JJ (2008) The effect of out-of-plane motion on 2D and 3D digital image correlation measurements. Opt Laser Eng 46(10):746-757

Sutton MA, Orteu JJ, Schreier H (2009) Image correlation for shape, motion and deformation measurements: basic concepts, theory and applications. Springer

Wei Q-Y, Lin H, Yang B, Li L, Zhang L-Q, Huang H-D, Zhong G-J, Xu L, Li Z-M (2020) Structure and properties of allcellulose composites prepared by controlling the dissolution temperature of a $\mathrm{NaOH} / \mathrm{Urea}$ solvent. Ind Eng Chem Res 59:10428-10435

Xu D, Cerbu C, Wang H, Rosca IC (2019) Analysis of the hybrid composite materials reinforced with natural fibers considering digital image correlation (DIC) measurements. Mech Mater 135:46-56

Publisher's Note Springer Nature remains neutral with regard to jurisdictional claims in published maps and institutional affiliations. 